\title{
Spectral-time analysis of relaxation and creep processes of polymeric materials
}

\author{
N. Pereborova \\ PhD, Associate Professor, Associate Professor of the Department of Intelligent Systems and Information \\ Security Saint Petersburg State University of Industrial Technologies and Design, Saint Petersburg, Russia \\ A. Makarov \\ Vice-Rector of Science, Saint Petersburg State University of Industrial Technologies and Design, Saint \\ Petersburg, Russia
}

A. Demidov

Rector, Saint Petersburg State University of Industrial Technologies and Design, Saint Petersburg, Russia

V. Wagner

PhD, Associate Professor of the Department of Intelligent Systems and Information Security Saint Petersburg State University of Industrial Technologies and Design, Saint Petersburg, Russia

\begin{abstract}
The article investigates into the type of distributions of relaxing and delay particles over their relaxation and delay times built upon the mathematical models of relaxation and creep of polymeric materials. The relaxation and delay times characterise the transition times of relaxing or delay particles from one stable energy state to another (Makarov et al., 2016).

The nature of such transitions can be different. It depends on both the rheology of a polymer material and the applied deformation or load value. It can be explained, on the one hand, by conformational energy transfers within the macromolecules of material when their shape changes during rearrangement, and on the other hand, there are shears of macromolecules and other changes caused by energy (Makarov et al., 2015a). The most satisfactory results considering the processes of deformation of polymeric materials can be obtained using rather complex models such as a consequent combination of a number of the Maxwell and the Kelvin-Voigt models (Pereborova et al., 2020a). The application of modelling for an accurate quantitative description of deformation or relaxation in the study of the properties of polymers encounters certain difficulties (Pereborova et al., 2020b). Different operational behaviour of polymers, structural changes during deformation depending on the sample background, temperature, duration of action, and stress-strain values, make it difficult to obtain the exact rheological characteristics of the process (Pereborova et al., 2020c). However, the relevance of modelling for a qualitative or approximate quantitative description of mechanical properties is obvious.
\end{abstract}

\section{THE METHODS FOR DETERMINING THE SPECTRA OF RELAXATION AND DELAY}

One of the methods for describing deformation and relaxation processes of polymer materials is the use of mechanical models. The limits and prospects of application of deformation processes modelling of polymers were considered in a number of studies carried out by V. A. Kargin (Pereborova et al., 2020d), G. L. Slonimskiy (Pereborova et al., 2020e), A .A. Askadskiy (Makarov et al., 2018a), I. Ward (Pereborova et al., 2019a) and other authors.

The study of relationships between various viscoelastic functions often deals with the relaxation and delay (creep) spectra. The essence of these spectra can be shown on the example of 
the Generalised Maxwell model (for relaxation) and on the example of the Generalised Kelvin-Voigt model (Pereborova et al., 2018a).

The Generalised Maxwell model represents an infinite parallel set of Maxwell elements consisting of a dashpot and a spring connected in series (Figure 1.).

Consider the element at the $i$-th place. If the stiffness of a spring is denoted by $E_{i}$, and the viscosity of the liquid where a dashpot $\eta_{i}$ is located, then the relaxation time of the $\mathrm{i}$-th element is obtained by $\tau_{i}=\frac{\eta_{i}}{E_{i}}$ and is a measure of the time required for stress relaxation.

Any number of Maxwell's elements connected in series has the properties of both the element itself and other more complex properties. This fact leads to a discrete spectrum of relaxation times $\left\{\tau_{i}\right\}$ (Pereborova et al., 2018b).

A similar statement can be made for the delay (creep) spectrum on the example of the Generalised Kelvin-Voigt model which is an infinite parallel set of Kelvin-Voigt elements consisting of a dashpot and a spring connected in series (Figure 2.) (Egorov et al., 2020).

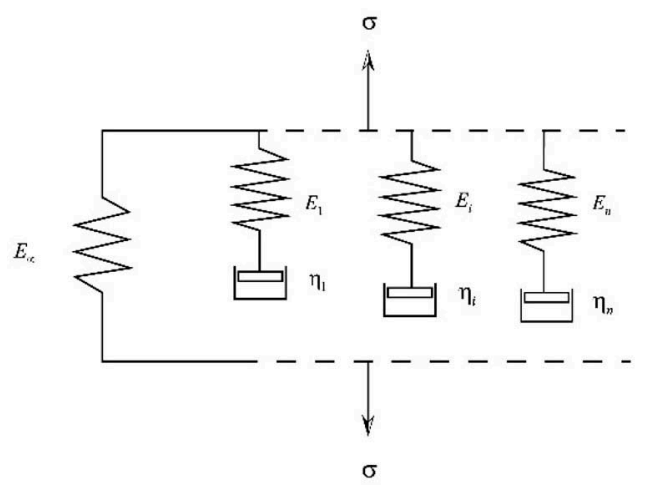

Figure 1. The Generalised Maxwell model.

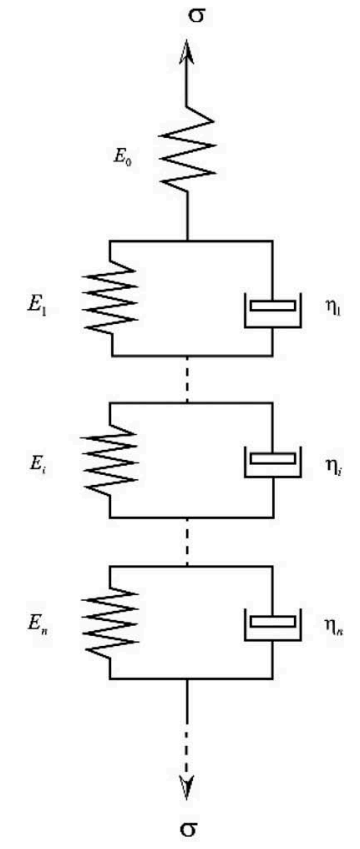

Figure 2. The Generalised Kelvin-Voigt model. 
If the element is at the $i$-th place consists of a spring with stiffness $E i$, and the viscosity of the liquid where a dashpot is located $\eta_{i}$, then the delay time of the $i$-th element is obtained by $\tau_{i}=\frac{\eta_{i}}{E_{i}}$ and is a measure of the time required for the spring to strain to its equilibrium length in the presence of the viscous element braking forces (Pereborova et al., 2019b).

\section{OBTAINING OF THE RELAXATION SPECTRA OF POLYMERIC MATERIALS}

Sufficient approximations of the relaxation and delay spectra can be found by processing the experimental sets of relaxation and creep, respectively. Obtaining the relaxation and delay spectra means finding the distribution of the relaxation times and delay times that cannot be found experimentally.

To obtain the analytical form of the relaxation spectrum of polymeric materials, a variant of the mathematical model of the relaxation process can be used in the form (Rymkevich et al., 2013).

$$
\sigma_{t}=E_{0} \varepsilon_{t}-\left(E_{0}-E_{\infty}\right) \cdot \int_{0}^{t} \varepsilon_{\theta} \cdot \varphi_{\varepsilon ; t-\theta}^{\prime} d \theta
$$

where

$\varepsilon_{t}$ - time-dependent deformation $t$,

$\sigma_{t}$ - time-dependent stress $t$,

$E_{0}$ - elastic modulus,

$E_{\infty}$ - viscoelastic modulus,

$$
\varphi_{\varepsilon t}=\frac{1}{2} \cdot\left(1+\operatorname{th}\left(\frac{A_{\varepsilon}}{2} \cdot \ln \left(\frac{t}{\tau_{\varepsilon}}\right)\right)\right)
$$

normalised relaxation function that forms the basis of the mathematical model of relaxation (Pereborova et al., 2019c),

$$
\begin{aligned}
& \varphi_{\varepsilon t}^{\prime}=\bar{r}_{\varepsilon t}=\frac{\partial \varphi_{\varepsilon t}}{\partial(\ln t)}=\frac{A_{\varepsilon}}{4} \cdot \frac{1}{c h^{2}\left(W_{\varepsilon t}\right)}= \\
& =\frac{A_{\varepsilon}}{4} \cdot\left(1-t h^{2}\left(W_{\varepsilon t}\right)\right)=A_{\varepsilon} \cdot \varphi_{\varepsilon t} \cdot\left(1-\varphi_{\varepsilon t}\right)
\end{aligned}
$$

integral relaxation kernel,

$$
W_{\varepsilon t}=\frac{A_{\varepsilon}}{2} \cdot \ln \frac{t}{\tau_{\varepsilon}}=\frac{A_{\varepsilon}}{2} \cdot\left(\ln \left(\frac{t}{t_{1}}\right)+\ln \left(\frac{t_{1}}{\tau_{\varepsilon}}\right)\right)
$$

$A_{\varepsilon}$ - parameter of the intensity of relaxation processes,

$$
f_{\varepsilon, \tau_{\varepsilon}}=\ln \left(\frac{t}{\tau_{\varepsilon}}\right)
$$

logarithmic function of the relaxation times.

The analytical form of the relaxation spectrum $\bar{H}_{\varepsilon \tilde{\tau}}$ can be obtained by the known formula (Pereborova et al., 2018c) 


$$
\int_{0}^{\infty} \bar{H}_{\varepsilon \tilde{\tau}} \cdot e^{-t x} \cdot d x=\frac{d \varphi_{\varepsilon t}}{d t}=r_{\varepsilon t}
$$

by successive approximations.

The given formula (6) is the Laplace transform in which the relaxation spectrum $\bar{H}_{\varepsilon \tilde{\tau}}$ plays the role of the original, and the relaxation kernel $r_{\varepsilon t}$ plays the role of the figure in terms of operator calculus. This dependence (6) is obtained on the basis of the properties of the Generalised Maxwell model (Pereborova et al., 2018d).

The application of the hyperbolic tangent function as the normalised relaxation function (2) leads to the following logarithmic relaxation kernel

$$
E_{\varepsilon t}^{\prime}=\frac{\partial E_{\varepsilon t}}{\partial \ln t}=-\left(E_{0}-E_{\infty}\right) \cdot \bar{r}_{\varepsilon t}=-\frac{A_{\varepsilon}}{4} \cdot\left(E_{0}-E_{\infty}\right) \cdot\left(1-t h^{2}\left(W_{\varepsilon t}\right)\right)
$$

The deformation-time argument (4) of the relaxation kernel (3) takes into account the current time factor and the characteristically average internal relaxation times. In the known approximation, the relaxation kernel (3) characterises the distribution of relaxing particles over their micromechanical relaxation times (Pereborova et al., 2020f).

In order to obtain approximations of the normalised relaxation spectrum, the known recurrence formulas can be used (Gorshkov et al., 2013a) if $k=1$

$$
\bar{H}_{1}=\frac{\partial \varphi_{\varepsilon \mathrm{t}}}{\partial \ln t}=\varphi_{\varepsilon \mathrm{t}}^{\prime}=\left.\bar{r}_{\varepsilon \mathrm{t}}\right|_{t=\tilde{\tau}}
$$

and if $k \quad 2$

$$
\bar{H}_{k}=\bar{H}_{k-1}-\left.\frac{1}{k-1} \cdot \frac{d \bar{H}_{k-1}}{d \ln \tilde{\tau}}\right|_{t=(k-1) \tilde{\tau}}
$$

For calculating the values of the derivatives $\bar{r}_{\varepsilon t}=\frac{\partial \varphi_{\varepsilon t}}{\partial \ln t}, \ldots, \frac{\partial^{k} \varphi_{\varepsilon t}}{\partial \ln t^{k}}, \ldots$, where for the relaxation function $\varphi_{\varepsilon t}=\varphi$ the hyperbolic tangent (2) is chosen and $\mathrm{W}_{\varepsilon \mathrm{t}}=W$ is the structuredeformation-time argument-functional (4), we obtain

$$
\begin{gathered}
\varphi_{\varepsilon t}=\frac{1}{2} \cdot\left(1+t h\left(\frac{A_{\varepsilon}}{2} \cdot \ln \left(\frac{t}{\tau_{\varepsilon}}\right)\right)\right) \\
\varphi_{\varepsilon t}^{\prime}=\bar{r}_{\varepsilon t}=\frac{\partial \varphi_{\varepsilon t}}{\partial(\ln t)}=\frac{A_{\varepsilon}}{4} \cdot \frac{1}{c h^{2}\left(W_{\varepsilon t}\right)}=\frac{A_{\varepsilon}}{4} \cdot\left(1-t h^{2}\left(W_{\varepsilon t}\right)\right)=A_{\varepsilon} \cdot \varphi_{\varepsilon t} \cdot\left(1-\varphi_{\varepsilon t}\right) \\
\varphi^{\prime \prime}{ }_{\varepsilon t}=\frac{\partial^{2} \varphi_{\varepsilon t}}{\partial(\ln t)^{2}}=A_{\varepsilon}^{2} \cdot \varphi_{\varepsilon t} \cdot\left(1-\varphi_{\varepsilon t}\right) \cdot\left(1-2 \cdot \varphi_{\varepsilon t}\right) \\
\varphi^{\prime \prime \prime}{ }_{\varepsilon t}=\frac{\partial^{3} \varphi_{\varepsilon t}}{\partial(\ln t)^{3}}=A_{\varepsilon}^{3} \cdot \varphi_{\varepsilon t} \cdot\left(1-\varphi_{\varepsilon t}\right) \cdot\left(1-6 \cdot \varphi_{\varepsilon t}+6 \cdot \varphi_{\varepsilon t}^{2}\right) \\
\varphi_{\varepsilon t}^{\iota v}=\frac{\partial^{4} \varphi_{\varepsilon t}}{\partial(\ln t)^{4}}=A_{\varepsilon}^{4} \cdot \varphi_{\varepsilon t} \cdot\left(1-\varphi_{\varepsilon t}\right) \times\left(1-14 \cdot \varphi_{\varepsilon t}+36 \cdot \varphi_{\varepsilon t}^{2}-24 \cdot \varphi_{\varepsilon t}^{3}\right) \\
\varphi_{\varepsilon t}^{v}=\frac{\partial^{5} \varphi_{\varepsilon t}}{\partial(\ln t)^{5}}=A_{\varepsilon}^{5} \cdot \varphi_{\varepsilon t} \cdot\left(1-\varphi_{\varepsilon t}\right) \times\left(1-30 \cdot \varphi_{\varepsilon t}+150 \cdot \varphi_{\varepsilon t}^{2}-240 \cdot \varphi_{\varepsilon t}^{3}+120 \cdot \varphi_{\varepsilon t}^{4}\right)
\end{gathered}
$$




$$
\begin{gathered}
\varphi_{\varepsilon t}^{v \iota}=\frac{\partial^{6} \varphi_{\varepsilon t}}{\partial(\ln t)^{6}}=A_{\varepsilon}^{6} \cdot \varphi_{\varepsilon t} \cdot\left(1-\varphi_{\varepsilon t}\right) \cdot\left(1-62 \cdot \varphi_{\varepsilon t}+\right. \\
\left.+510 \cdot \varphi_{\varepsilon t}^{2}-1560 \cdot \varphi_{\varepsilon t}^{3}+1800 \cdot \varphi_{\varepsilon t}^{4}-720 \cdot \varphi_{\varepsilon t}^{5}\right) \\
\varphi_{\varepsilon t}^{v v l}=\frac{\partial^{7} \varphi_{\varepsilon t}}{\partial(\ln t)^{7}}=A_{\varepsilon}^{6} \cdot \varphi_{\varepsilon t} \cdot\left(1-\varphi_{\varepsilon t}\right) \cdot\left(1-126 \cdot \varphi_{\varepsilon t}+1716 \cdot \varphi_{\varepsilon t}^{2}-\right. \\
\left.-8280 \cdot \varphi_{\varepsilon t}^{3}+16800 \cdot \varphi_{\varepsilon t}^{4}-15120 \cdot \varphi_{\varepsilon t}^{5}+5040 \cdot \varphi_{\varepsilon t}^{6}\right)
\end{gathered}
$$

Using the recurrence formulas (8), (9) that in expanded form are given by

$$
\begin{gathered}
\bar{H}_{1}=\frac{\partial \varphi}{\partial \ln t}=\varphi^{\prime}=\left.\overline{r_{\varepsilon t}}\right|_{t=\tilde{\tau}} \\
\bar{H}_{2}=\frac{\partial \varphi}{\partial \ln t}-\frac{\partial^{2} \varphi}{\partial \ln t^{2}}=\varphi^{\prime}-\left.\varphi^{\prime \prime}\right|_{t=\tilde{\tau}} \\
\bar{H}_{3}=\varphi^{\prime}-\frac{3}{2} \varphi^{\prime \prime}+\left.\frac{1}{2} \varphi^{\prime \prime \prime}\right|_{t=2 \tilde{\tau}} \\
\bar{H}_{4}=\varphi^{\prime}-\frac{11}{6} \varphi^{\prime \prime}+\varphi^{\prime \prime \prime}-\left.\frac{1}{6} \varphi^{\iota v}\right|_{t=3 \tilde{\tau}} \\
\bar{H}_{5}=\varphi^{\prime}-\frac{25}{12} \varphi^{\prime \prime}+\frac{35}{24} \varphi^{\prime \prime \prime}-\frac{5}{12} \varphi^{\iota v}+\left.\frac{1}{24} \varphi^{v}\right|_{t=4 \tilde{\tau}} \\
\bar{H}_{6}=\varphi^{\prime}-\frac{137}{60} \varphi^{\prime \prime}+\frac{15}{8} \varphi^{\prime \prime \prime}-\frac{17}{24} \varphi^{\iota v}+\frac{3}{24} \varphi^{v}-\left.\frac{1}{120} \varphi^{v \iota}\right|_{t=5 \tilde{\tau}} \\
\bar{H}_{7}=\varphi^{\prime}-\frac{49}{20} \varphi^{\prime \prime}+\frac{203}{90} \varphi^{\prime \prime \prime}- \\
-\frac{49}{48} \varphi^{\iota v}+\frac{35}{144} \varphi^{v}-\frac{7}{240} \varphi^{v \iota}+\left.\frac{1}{720} \varphi^{v \iota l}\right|_{t=6 \tilde{\tau}}
\end{gathered}
$$

we obtain expressions for approximations of the normalised relaxation spectrum

$$
\begin{aligned}
& \bar{H}_{1}=\left.\frac{\partial \varphi_{\varepsilon t}}{\partial(\ln t)}\right|_{t=\tilde{\tau}}=\left.\varphi_{\varepsilon t}^{\prime}\right|_{t=\tilde{\tau}}=\left.A_{\varepsilon} \cdot \varphi_{\varepsilon t} \cdot\left(1-\varphi_{\varepsilon t}\right)\right|_{t=\tilde{\tau}} \\
& \bar{H}_{2}=\varphi_{\varepsilon t}^{\prime}-\left.\varphi^{\prime \prime}\right|_{\varepsilon t=\tilde{\tau}}=\left.A_{\varepsilon} \cdot \varphi_{\varepsilon t} \cdot\left(1-\varphi_{\varepsilon t}\right) \cdot\left(1-A_{\varepsilon}\left(1-2 \cdot \varphi_{\varepsilon t}\right)\right)\right|_{t=\tilde{\tau}} \\
& \bar{H}_{3}=\varphi_{\varepsilon t}^{\prime}-\frac{3}{2} \varphi^{\prime \prime}{ }_{\varepsilon t}+\left.\frac{1}{2} \varphi^{\prime \prime \prime}{ }_{\varepsilon t}\right|_{t=2 \tilde{\tau}}=A_{\varepsilon} \cdot \varphi_{\varepsilon t} \cdot\left(1-\varphi_{\varepsilon t}\right) \times \\
& \times\left.\left(1-\frac{3}{2} \cdot A_{\varepsilon} \cdot\left(1-2 \cdot \varphi_{\varepsilon t}\right)+\frac{1}{2} \cdot A_{\varepsilon}^{2} \cdot\left(1-6 \cdot \varphi_{\varepsilon t}+6 \cdot \varphi_{\varepsilon t}^{2}\right)\right)\right|_{t=2 \tilde{\tau}} \\
& \bar{H}_{4}=\varphi_{\varepsilon t}^{\prime}-\frac{11}{6} \varphi^{\prime \prime}{ }_{\varepsilon t}+\varphi^{\prime \prime \prime}{ }_{\varepsilon t}-\left.\frac{1}{6} \varphi^{\prime \prime \prime \prime}{ }_{\varepsilon t}\right|_{t=3 \tilde{\tau}}=A_{\varepsilon} \cdot \varphi_{\varepsilon t} \cdot\left(1-\varphi_{\varepsilon t}\right) \times \\
& \times\left(1-\frac{11}{6} \cdot A_{\varepsilon} \cdot\left(1-2 \cdot \varphi_{\varepsilon t}\right)+A_{\varepsilon}^{2} \cdot\left(1-6 \cdot \varphi_{\varepsilon t}+6 \cdot \varphi_{\varepsilon t}^{2}\right)-\right. \\
& \left.-\frac{1}{6} \cdot A_{\varepsilon}^{3} \cdot\left(1-14 \cdot \varphi_{\varepsilon t}+36 \cdot \varphi_{\varepsilon t}^{2}-24 \cdot \varphi_{\varepsilon t}^{3}\right)\right)\left.\right|_{t=3 \tilde{\tau}}
\end{aligned}
$$




$$
\begin{aligned}
& \bar{H}_{5}=\varphi_{\varepsilon t}^{\prime}-\frac{25}{12} \varphi_{\varepsilon t}^{\prime \prime}+\frac{35}{24} \varphi^{\prime \prime \prime}{ }_{\varepsilon t}-\frac{5}{12} \varphi_{\varepsilon t}^{\prime \prime \prime \prime}+\left.\frac{1}{24} \varphi^{\prime \prime \prime \prime \prime}\right|_{t=4 \tilde{\tau}}= \\
& =A_{\varepsilon} \cdot \varphi_{\varepsilon t} \cdot\left(1-\varphi_{\varepsilon t}\right) \cdot\left(1-\frac{25}{12} \cdot A_{\varepsilon} \cdot\left(1-2 \cdot \varphi_{\varepsilon t}\right)+\frac{35}{24} \cdot A_{\varepsilon}^{2} \times\right. \\
& \times\left(1-6 \cdot \varphi_{\varepsilon t}+6 \cdot \varphi_{\varepsilon t}^{2}\right)-\frac{5}{12} \cdot A_{\varepsilon}^{3} \cdot\left(1-14 \cdot \varphi_{\varepsilon t}+36 \cdot \varphi_{\varepsilon t}^{2}-24 \cdot \varphi_{\varepsilon t}^{3}\right)+ \\
& \left.+\frac{1}{24} \cdot A_{\varepsilon}^{4} \cdot\left(1-30 \cdot \varphi_{\varepsilon t}+150 \cdot \varphi_{\varepsilon t}^{2}-240 \cdot \varphi_{\varepsilon t}^{3}+120 \cdot \varphi_{\varepsilon t}^{4}\right)\right)\left.\right|_{t=4 \tilde{\tau}} \\
& \bar{H}_{6}=\varphi_{\varepsilon t}^{\prime}-\frac{137}{60} \varphi^{\prime \prime}{ }_{\varepsilon t}+\frac{15}{8} \varphi^{\prime \prime \prime}{ }_{\varepsilon t}-\frac{17}{24} \varphi_{\varepsilon t}^{\iota v}+\frac{1}{8} \varphi_{\varepsilon t}^{v}-\left.\frac{1}{120} \varphi_{\varepsilon t}^{v \iota}\right|_{t=5 \tilde{\tau}}= \\
& =A_{\varepsilon} \cdot \varphi_{\varepsilon t} \cdot\left(1-\varphi_{\varepsilon t}\right) \cdot\left(1-\frac{137}{60} \cdot A_{\varepsilon} \cdot\left(1-2 \cdot \varphi_{\varepsilon t}\right)+\frac{15}{8} \cdot A_{\varepsilon}^{2} \times\right. \\
& \times\left(1-6 \cdot \varphi_{\varepsilon t}+6 \cdot \varphi_{\varepsilon t}^{2}\right)-\frac{17}{24} \cdot A_{\varepsilon}^{3} \cdot\left(1-14 \cdot \varphi_{\varepsilon t}+36 \cdot \varphi_{\varepsilon t}^{2}-24 \cdot \varphi_{\varepsilon t}^{3}\right)+ \\
& +\frac{1}{8} \cdot A_{\varepsilon}^{4} \cdot\left(1-30 \cdot \varphi_{\varepsilon t}+150 \cdot \varphi_{\varepsilon t}^{2}-240 \cdot \varphi_{\varepsilon t}^{3}+120 \cdot \varphi_{\varepsilon t}^{4}\right)-\frac{1}{120} \cdot A_{\varepsilon}^{5} \times \\
& \left.\times\left(1-62 \cdot \varphi_{\varepsilon t}+510 \cdot \varphi_{\varepsilon t}^{2}-1560 \cdot \varphi_{\varepsilon t}^{3}+1800 \cdot \varphi_{\varepsilon t}^{4}-720 \cdot \varphi_{\varepsilon t}^{5}\right)\right)\left.\right|_{t=5 \tau} \\
& \bar{H}_{7}=\varphi_{\varepsilon t}^{\prime}-\frac{49}{20} \varphi_{\varepsilon t}^{\prime \prime}+\frac{203}{90} \varphi_{\varepsilon t}^{\prime \prime \prime}-\frac{49}{48} \varphi_{\varepsilon t}^{\iota v}+\frac{35}{144} \varphi_{\varepsilon t}^{v}-\frac{7}{240} \varphi_{\varepsilon t}^{v l}+ \\
& +\left.\frac{1}{720} \varphi_{\varepsilon t}^{v u}\right|_{t=6 \tilde{\tau}}=A_{\varepsilon} \cdot \varphi_{\varepsilon t} \cdot\left(1-\varphi_{\varepsilon t}\right) \cdot\left(1-\frac{49}{20} \cdot A_{\varepsilon} \cdot\left(1-2 \cdot \varphi_{\varepsilon t}\right)+\right. \\
& +\frac{203}{90} \cdot A_{\varepsilon}^{2} \cdot\left(1-6 \cdot \varphi_{\varepsilon t}+6 \cdot \varphi_{\varepsilon t}^{2}\right)-\frac{49}{48} \cdot A_{\varepsilon}^{3} \cdot\left(1-14 \cdot \varphi_{\varepsilon t}+36 \cdot \varphi_{\varepsilon t}^{2}-\right. \\
& \left.-24 \cdot \varphi_{\varepsilon t}^{3}\right)+\frac{35}{144} \cdot A_{\varepsilon}^{4} \cdot\left(1-30 \cdot \varphi_{\varepsilon t}+150 \cdot \varphi_{\varepsilon t}^{2}-240 \cdot \varphi_{\varepsilon t}^{3}+120 \cdot \varphi_{\varepsilon t}^{4}\right)- \\
& -\frac{7}{240} \cdot A_{\varepsilon}^{5} \cdot\left(1-62 \cdot \varphi_{\varepsilon t}+510 \cdot \varphi_{\varepsilon t}^{2}-1560 \cdot \varphi_{\varepsilon t}^{3}+\right. \\
& \left.+1800 \cdot \varphi_{\varepsilon t}^{4}-720 \cdot \varphi_{\varepsilon t}^{5}\right)+\frac{1}{720} \cdot A_{\varepsilon}^{6} \cdot\left(1-126 \cdot \varphi_{\varepsilon t}+1760 \cdot \varphi_{\varepsilon t}^{2}-\right. \\
& \left.\left.-8280 \cdot \varphi_{\varepsilon t}^{3}+16800 \cdot \varphi_{\varepsilon t}^{4}-15120 \cdot \varphi_{\varepsilon t}^{5}+5040 \cdot \varphi_{\varepsilon t}^{6}\right)\right)\left.\right|_{t=6 \tilde{\tau}}
\end{aligned}
$$

The obtained results (25) - (31) can be represented by a single formula (Makarov et al., 2017a).

$$
\bar{H}_{k}=\left.\bar{r}_{\varepsilon t} \cdot \sum_{i=1}^{k}\left(A_{\varepsilon}^{i-1} \cdot P_{k i}\left(\varphi_{\varepsilon t}\right)\right)\right|_{t=(k-1) \tilde{\tau}}
$$

where $P_{k i}\left(\varphi_{\varepsilon t}\right)$ - polynomials of a degree $i-1$ from $\varphi_{\varepsilon t}$. The type of the polynomials $P_{k i}\left(\varphi_{\varepsilon t}\right) \leftarrow$ is shown in Table 1.

Therefore, the relaxation spectrum obtained on the basis of the mathematical model of the relaxation process (1) using the hyperbolic tangent (2) as the normalised relaxation function is completely determined by the structural coefficient $A_{\varepsilon}$ depending on a polymer material and a type of the polynomials $P_{k i}\left(\varphi_{\varepsilon t}\right)$ that do not depend on a polymer material but are characteristics of the mathematical model itself (Demidov et al., 2017).

From the analytical form of the relaxation spectrum approximations $\bar{H}_{k}$, it is evident that these approximations converge rather quickly to their limiting value $\bar{H}$ which characterises the stability of the chosen mathematical model of the relaxation process (1) based on the 
Table 1. The type of polynomials $P_{k i}\left(\varphi_{\varepsilon t}\right)$ included in the formulas for approximations of the relaxation spectra of polymer materials.

\begin{tabular}{llllll}
\hline $\mathrm{i} \mid \mathrm{k}$ & 1 & 2 & 3 & 4 & 5 \\
\hline 1 & 1 & 1 & 1 & 1 & 1 \\
2 & - & $2 \varphi-1$ & $3 \varphi-\frac{3}{2}$ & $\frac{11}{3} \varphi-\frac{11}{6}$ & $\frac{25}{6} \varphi-\frac{25}{12}$ \\
3 & - & - & $3 \varphi^{2}-3 \varphi+\frac{1}{2}$ & $6 \varphi^{2}-6 \varphi+1$ & $\frac{35}{4} \varphi^{2}-\frac{35}{4} \varphi+\frac{35}{24}$ \\
4 & - & - & - & $4 \varphi^{3}-6 \varphi^{2}+\frac{7}{3} \varphi-\frac{1}{6}$ & $10 \varphi^{3}-15 \varphi^{2}+\frac{35}{6} \varphi-\frac{5}{12}$ \\
5 & - & - & - & - & $5 \varphi^{4}-10 \varphi^{3}+\frac{25}{4} \varphi^{2}-\frac{5}{4} \varphi+\frac{1}{24}$ \\
\hline
\end{tabular}

\begin{tabular}{lll}
\hline ilk & 6 & 7 \\
\hline 1 & 1 & 1 \\
2 & $\frac{137}{30} \varphi-\frac{137}{60}$ & $\frac{49}{10} \varphi-\frac{49}{20}$ \\
3 & $\frac{45}{4} \varphi^{2}-\frac{45}{4} \varphi+\frac{15}{8}$ & $\frac{203}{15} \varphi^{2}-\frac{203}{15} \varphi+\frac{203}{90}$ \\
4 & $17 \varphi^{3}-\frac{51}{2} \varphi^{2}+\frac{119}{12} \varphi-\frac{17}{24}$ & $\frac{49}{2} \varphi^{3}-\frac{147}{4} \varphi^{2}+\frac{343}{24} \varphi-\frac{49}{48}$ \\
5 & $15 \varphi^{4}-30 \varphi^{3}+\frac{75}{4} \varphi^{2}-\frac{15}{4} \varphi+\frac{1}{8}$ & $\frac{175}{6} \varphi^{4}-\frac{175}{3} \varphi^{3}+\frac{875}{24} \varphi^{2}-\frac{175}{24} \varphi+\frac{35}{144}$ \\
6 & $6 \varphi^{5}-15 \varphi^{4}+13 \varphi^{3}-\frac{17}{4} \varphi^{2}+\frac{31}{60} \varphi-\frac{1}{120}$ & $21 \varphi^{5}-\frac{105}{2} \varphi^{4}+\frac{91}{2} \varphi^{3}-$ \\
7 & & $-\frac{119}{8} \varphi^{2}+\frac{217}{120} \varphi-\frac{7}{240}$ \\
& - & $7 \varphi^{6}-21 \varphi^{5}+\frac{70}{3} \varphi^{4}-\frac{23}{2} \varphi^{3}+$ \\
\hline
\end{tabular}

hyperbolic tangent (2). This means that, with a small permissible error, as the relaxation spectrum its first approximation can be accepted, which is given by (Makarov et al., 2017b).

$$
\bar{H}_{1}=\left.A_{\varepsilon} \cdot \varphi_{\varepsilon t} \cdot\left(1-\varphi_{\varepsilon t}\right)\right|_{t=\tilde{\tau}}
$$

and depends only on the structural coefficient $A_{\varepsilon}$ and on the relaxation function $\varphi_{\varepsilon t}$ (2).

Taking into account that the approximations of the spectrum of relaxation times $\bar{H}_{\varepsilon \tilde{\tau}}$ are obtained by the logarithmic time scale derivatives of the normalised relaxation function (2) and knowing the type of the relaxation spectrum, the inverse problem can be solved. It is possible to obtain the corresponding normalised relaxation function by integrating. That is the basis of the mathematical model of viscoelastic properties of polymeric materials (Pereborova, $2020 \mathrm{~g}$ ).

The example of the relaxation spectrum of a polyester thread 83 tex. is shown in Figure 3 . And the corresponding spectrum of the relaxation times of a polyester fabric made from polyester threads 83 tex. is shown in Figure 4.

Analysing the graphs of the relaxation times spectra of a polyester thread 83 tex. and a polyester fabric made of polyester threads 83 tex., it can be noted that the intensity parameter corresponds to a polyester thread $A_{\varepsilon}=0,14$, while in a polyester fabric it is much higher $A_{\varepsilon}=0,92$ which leads to a more pronounced shape of the relaxation spectrum curve (Figure 3 ., Figure 4.) (Makarov et al., 2015b).

It should be noted that the modified logarithmic time scale contains the deformation-time function

$$
\ln \frac{t}{\tau_{\varepsilon}}=\ln \frac{t}{t_{1}}+\ln \frac{t_{1}}{\tau_{\varepsilon}}
$$




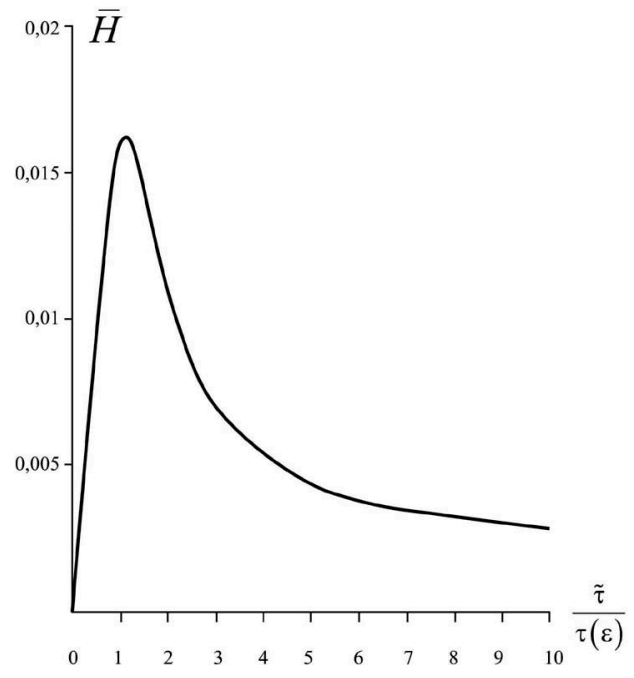

Figure 3. The graph of the spectrum of relaxation times of a polyester thread 83 tex., $T=40^{\circ} \mathrm{C}$.

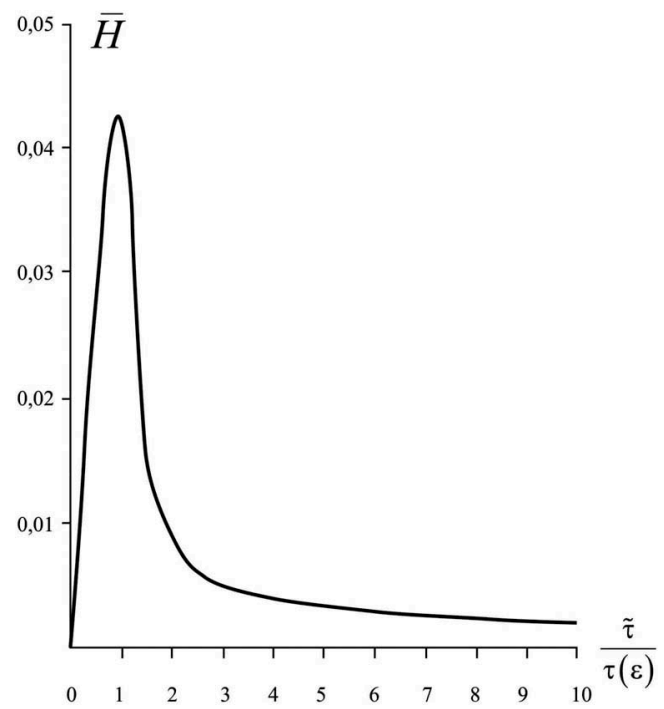

Figure 4. The graph of the spectrum of relaxation times of polyester fabric for technical purposes, $T=40^{\circ} \mathrm{C}$.

reflecting the deformation-time analogy. Due to this time modification, the obtained relaxation spectra are generalised, that means, they extend to the entire initial range of nondestructive deformation (Makarov et al., 2014a).

\section{OBTAINING OF THE DELAY SPECTRA OF POLYMERIC MATERIALS}

To obtain the analytical form of the delay spectrum of polymeric materials, a variant of the mathematical model of the creep process can be used in the form (Demidov et al., 2006a). 
where

$$
\varepsilon_{t}=D_{0} \sigma_{t}+\left(D_{\infty}-D_{0}\right) \cdot \int_{0}^{t} \sigma_{\theta} \cdot \varphi_{\sigma ; t-\theta}^{\prime} d \theta
$$

$\varepsilon_{t}$ - time-dependent deformation $t$,

$\sigma_{t}$ - time-dependent stress $t$,

$D_{0}$ - initial elastic compliance,

$D_{\infty}$ - finite quasiequilibrium compliance,

$$
\varphi_{\sigma t}=\frac{1}{2} \cdot\left(1+\operatorname{th}\left(\frac{A_{\sigma}}{2} \cdot \ln \left(\frac{t}{\tau_{\sigma}}\right)\right)\right)
$$

normalised delay function that forms the basis of the mathematical model of creep (Demidov et al., 2009),

$$
\varphi_{\sigma t}^{\prime}=\bar{r}_{\sigma t}=\frac{\partial \varphi_{\sigma t}}{\partial(\ln t)}=\frac{A_{\sigma}}{4} \cdot \frac{1}{c h^{2}\left(W_{\sigma t}\right)}=\frac{A_{\sigma}}{4} \cdot\left(1-t h^{2}\left(W_{\sigma t}\right)\right)=A_{\sigma} \cdot \varphi_{\sigma t} \cdot\left(1-\varphi_{\sigma t}\right)
$$

integral delay kernel,

$$
W_{\sigma t}=\frac{A_{\sigma}}{2} \cdot \ln \frac{t}{\tau_{\sigma}}=\frac{A_{\sigma}}{2} \cdot\left(\ln \left(\frac{t}{t_{1}}\right)+\ln \left(\frac{t_{1}}{\tau_{\sigma}}\right)\right)
$$

$A_{\sigma}$ - parameter of the intensity of creep processes,

$$
f_{\sigma, \tau_{\sigma}}=\ln \left(\frac{t}{\tau_{\sigma}}\right)
$$

logarithmic function of the delay times.

The analytical form of the delay spectrum $\bar{Q}_{\sigma \tilde{\tau}}$ can be obtained by the known formula (Makarov et al., 2014b).

$$
\int_{0}^{\infty} \bar{Q}_{\sigma \tilde{\tau}} \cdot e^{-t x} \cdot d x=\frac{d \varphi_{\sigma t}}{d t}=r_{\sigma t}
$$

by successive approximations.

The given formula (41) is the Laplace transform in which the delay spectrum $\bar{Q}_{\sigma \tilde{\tau}}$ plays the role of the original, and the delay kernel $r_{\sigma t}$ plays the role of the figure in terms of operator calculus. This dependence (40) is obtained on the basis of the properties of the Generalised Kelvin-Voigt model (Demidov et al., 2007a).

The application of the hyperbolic tangent function as the normalised delay function (36), leads to the following logarithmic delay kernel (Makarov et al., 2020).

$$
D_{\sigma t}^{\prime}=\frac{\partial D_{\sigma t}}{\partial \ln t}=\left(D_{\infty}-D_{0}\right) \cdot \bar{r}_{\sigma t}=\frac{A_{\sigma}}{4} \cdot\left(D_{\infty}-D_{0}\right) \cdot\left(1-t h^{2}\left(W_{\sigma t}\right)\right)
$$

The force-time argument (38) of the delay kernel (37) takes into account the current time factor and the characteristically average internal delay times. In the known approximation, the delay kernel (37) characterises the distribution of relaxing particles over their micromechanical delay times (Demidov et al., 2006b). 
In order to obtain approximations of the normalised delay spectrum, the known recurrence formulas can be used (Demidov et al., 2006c) if $k=1$

$$
\bar{Q}_{1}=\frac{\partial \varphi_{\sigma \mathrm{t}}}{\partial \ln t}=\varphi_{\sigma \mathrm{t}}^{\prime}=\left.\bar{r}_{\sigma \mathrm{t}}\right|_{t=\tilde{\tau}}
$$

and if $k 2$

$$
\bar{Q}_{k}=\bar{Q}_{k-1}-\left.\frac{1}{k-1} \cdot \stackrel{d \bar{Q}_{k-1}}{d \ln \tilde{\tau}}\right|_{t=(k-1) \tilde{\tau}}
$$

For calculating the values of the derivatives $\bar{r}_{\sigma t}=\frac{\partial \varphi_{\sigma t}}{\partial \ln t}, \ldots, \frac{\partial^{k} \varphi_{\sigma t}}{\partial \ln t^{k}}, \ldots$, where for the delay function $\varphi_{\sigma t}=\varphi$ the hyperbolic tangent is chosen (36) and $\mathrm{W}_{\sigma \mathrm{t}}=W$ is the structure-force-time argument-functional (38), we obtain

$$
\begin{aligned}
& \varphi_{\sigma t}=\frac{1}{2} \cdot\left(1+t h\left(\frac{A_{\sigma}}{2} \cdot \ln \left(\frac{t}{\tau_{\sigma}}\right)\right)\right) \\
& \varphi_{\sigma t}^{\prime}=\bar{r}_{\sigma t}=\frac{\partial \varphi_{\sigma t}}{\partial(\ln t)}=\frac{A_{\sigma}}{4} \cdot \frac{1}{c h^{2}\left(W_{\sigma t}\right)}= \\
& =\frac{A_{\sigma}}{4} \cdot\left(1-t h^{2}\left(W_{\sigma t}\right)\right)=A_{\sigma} \cdot \varphi_{\sigma t} \cdot\left(1-\varphi_{\sigma t}\right) \\
& \varphi^{\prime \prime}{ }_{\sigma t}=\frac{\partial^{2} \varphi_{\sigma t}}{\partial(\ln t)^{2}}=A_{\sigma}^{2} \cdot \varphi_{\sigma t} \cdot\left(1-\varphi_{\sigma t}\right) \cdot\left(1-2 \cdot \varphi_{\sigma t}\right) \\
& \varphi^{\prime \prime \prime}{ }_{\sigma t}=\frac{\partial^{3} \varphi_{\sigma t}}{\partial(\ln t)^{3}}=A_{\sigma}^{3} \cdot \varphi_{\sigma t} \cdot\left(1-\varphi_{\sigma t}\right) \cdot\left(1-6 \cdot \varphi_{\sigma t}+6 \cdot \varphi_{\sigma t}^{2}\right) \\
& \varphi_{\sigma t}^{\iota v}=\frac{\partial^{4} \varphi_{\sigma t}}{\partial(\ln t)^{4}}=A_{\sigma}^{4} \cdot \varphi_{\sigma t} \cdot\left(1-\varphi_{\sigma t}\right) \times \\
& \times\left(1-14 \cdot \varphi_{\sigma t}+36 \cdot \varphi_{\sigma t}^{2}-24 \cdot \varphi_{\sigma t}^{3}\right) \\
& \varphi_{\sigma t}^{v}=\frac{\partial^{5} \varphi_{\sigma t}}{\partial(\ln t)^{5}}=A_{\sigma}^{5} \cdot \varphi_{\sigma t} \cdot\left(1-\varphi_{\sigma t}\right) \times \\
& \times\left(1-30 \cdot \varphi_{\sigma t}+150 \cdot \varphi_{\sigma t}^{2}-240 \cdot \varphi_{\sigma t}^{3}+120 \cdot \varphi_{\sigma t}^{4}\right) \\
& \varphi_{\sigma t}^{v \iota}=\frac{\partial^{6} \varphi_{\sigma t}}{\partial(\ln t)^{6}}=A_{\sigma}^{6} \cdot \varphi_{\sigma t} \cdot\left(1-\varphi_{\sigma t}\right) \cdot\left(1-62 \cdot \varphi_{\sigma t}+\right. \\
& \left.+510 \cdot \varphi_{\sigma t}^{2}-1560 \cdot \varphi_{\sigma t}^{3}+1800 \cdot \varphi_{\sigma t}^{4}-720 \cdot \varphi_{\sigma t}^{5}\right) \\
& \varphi_{\sigma t}^{v u \iota}=\frac{\partial^{7} \varphi_{\sigma t}}{\partial(\ln t)^{7}}=A_{\sigma}^{6} \cdot \varphi_{\sigma t} \cdot\left(1-\varphi_{\sigma t}\right) \cdot\left(1-126 \cdot \varphi_{\sigma t}+1716 \cdot \varphi_{\sigma t}^{2}-\right. \\
& \left.-8280 \cdot \varphi_{\sigma t}^{3}+16800 \cdot \varphi_{\sigma t}^{4}-15120 \cdot \varphi_{\sigma t}^{5}+5040 \cdot \varphi_{\sigma t}^{6}\right)
\end{aligned}
$$

and using the recurrence formulas (42), (43), that in expanded form are given by 


$$
\begin{gathered}
\bar{Q}_{1}=\frac{\partial \varphi}{\partial \ln t}=\varphi^{\prime}=\left.\overline{r_{\sigma \tau}}\right|_{t=\tilde{\tau}} \\
\bar{Q}_{2}=\frac{\partial \varphi}{\partial \ln t}-\frac{\partial^{2} \varphi}{\partial \ln t^{2}}=\varphi^{\prime}-\left.\varphi^{\prime \prime}\right|_{t=\tilde{\tau}} \\
\bar{Q}_{3}=\varphi^{\prime}-\frac{3}{2} \varphi^{\prime \prime}+\left.\frac{1}{2} \varphi^{\prime \prime \prime}\right|_{t=2 \tilde{\tau}} \\
\bar{Q}_{4}=\varphi^{\prime}-\frac{11}{6} \varphi^{\prime \prime}+\varphi^{\prime \prime \prime}-\left.\frac{1}{6} \varphi^{\iota v}\right|_{t=3 \tilde{\tau}} \\
\bar{Q}_{5}=\varphi^{\prime}-\frac{25}{12} \varphi^{\prime \prime}+\frac{35}{24} \varphi^{\prime \prime \prime}-\frac{5}{12} \varphi^{\iota v}+\left.\frac{1}{24} \varphi^{v}\right|_{t=4 \tilde{\tau}} \\
\bar{Q}_{6}=\varphi^{\prime}-\frac{137}{60} \varphi^{\prime \prime}+\frac{15}{8} \varphi^{\prime \prime \prime}-\frac{17}{24} \varphi^{\iota v}+\frac{3}{24} \varphi^{v}-\left.\frac{1}{120} \varphi^{v \iota}\right|_{t=5 \tilde{\tau}} \\
\bar{Q}_{7}=\varphi^{\prime}-\frac{49}{20} \varphi^{\prime \prime}+\frac{203}{90} \varphi^{\prime \prime \prime}-\frac{49}{48} \varphi^{\iota v}+\frac{35}{144} \varphi^{v}-\frac{7}{240} \varphi^{v \iota}+\left.\frac{1}{720} \varphi^{v \iota \iota}\right|_{t=6 \tilde{\tau}}
\end{gathered}
$$

we obtain expressions for approximations of the normalised delay spectrum

$$
\begin{gathered}
\bar{Q}_{1}=\left.\frac{\partial \varphi_{\sigma t}}{\partial(\ln t)}\right|_{t=\tilde{\tau}}=\left.\varphi_{\sigma t}^{\prime}\right|_{t=\tilde{\tau}}=\left.A_{\sigma} \cdot \varphi_{\sigma t} \cdot\left(1-\varphi_{\sigma t}\right)\right|_{t=\tilde{\tau}} \\
\bar{Q}_{2}=\varphi_{\sigma t}^{\prime}-\left.\varphi^{\prime \prime}{ }_{\sigma t}\right|_{t=\tilde{\tau}}=\left.A_{\sigma} \cdot \varphi_{\sigma t} \cdot\left(1-\varphi_{\sigma t}\right) \cdot\left(1-A_{\sigma}\left(1-2 \cdot \varphi_{\sigma t}\right)\right)\right|_{t=\tilde{\tau}} \\
\bar{Q}_{3}=\varphi_{\sigma t}^{\prime}-\frac{3}{2} \varphi^{\prime \prime}{ }_{\sigma t}+\left.\frac{1}{2} \varphi^{\prime \prime \prime}{ }_{\sigma t}\right|_{t=2 \tilde{\tau}}=A_{\sigma} \cdot \varphi_{\sigma t} \cdot\left(1-\varphi_{\sigma t}\right) \times \\
\times\left.\left(1-\frac{3}{2} \cdot A_{\sigma} \cdot\left(1-2 \cdot \varphi_{\sigma t}\right)+\frac{1}{2} \cdot A_{\sigma}^{2} \cdot\left(1-6 \cdot \varphi_{\sigma t}+6 \cdot \varphi_{\sigma t}^{2}\right)\right)\right|_{t=2 \tilde{\tau}} \\
\bar{Q}_{4}=\varphi^{\prime}{ }_{\sigma t}-\frac{11}{6} \varphi^{\prime \prime}{ }_{\sigma t}+\varphi^{\prime \prime \prime}{ }_{\sigma t}-\left.\frac{1}{6} \varphi^{\prime \prime \prime \prime}{ }_{\sigma t}\right|_{t=3 \tilde{\tau}}=A_{\sigma} \cdot \varphi_{\sigma t} \cdot\left(1-\varphi_{\sigma t}\right) \times \\
\times\left(1-\frac{11}{6} \cdot A_{\sigma} \cdot\left(1-2 \cdot \varphi_{\sigma t}\right)+A_{\sigma}^{2} \cdot\left(1-6 \cdot \varphi_{\sigma t}+6 \cdot \varphi_{\sigma t}^{2}\right)-\right. \\
\left.-\frac{1}{6} \cdot A_{\sigma}^{3} \cdot\left(1-14 \cdot \varphi_{\sigma t}+36 \cdot \varphi_{\sigma t}^{2}-24 \cdot \varphi_{\sigma t}^{3}\right)\right)\left.\right|_{t=3 \tilde{\tau}} \\
\bar{Q}_{5}=\varphi_{\sigma t}^{\prime}-\frac{25}{12} \varphi_{\sigma t}^{\prime \prime}+\frac{35}{24} \varphi^{\prime \prime \prime}{ }_{\sigma t}-\frac{5}{12} \varphi^{\prime \prime \prime \prime}{ }_{\sigma t}+\left.\frac{1}{24} \varphi^{\prime \prime \prime \prime \prime}{ }_{\sigma t}\right|_{t=4 \tilde{\tau}}= \\
=A_{\sigma} \cdot \varphi_{\sigma t} \cdot\left(1-\varphi_{\sigma t}\right) \cdot\left(1-\frac{25}{12} \cdot A_{\sigma} \cdot\left(1-2 \cdot \varphi_{\sigma t}\right)+\frac{35}{24} \cdot A_{\sigma}^{2} \times\right. \\
\times\left(1-6 \cdot \varphi_{\sigma t}+6 \cdot \varphi_{\sigma t}^{2}\right)-\frac{5}{12} \cdot A_{\sigma}^{3} \cdot\left(1-14 \cdot \varphi_{\sigma t}+36 \cdot \varphi_{\sigma t}^{2}-24 \cdot \varphi_{\sigma t}^{3}\right)+ \\
\left.\frac{1}{24} \cdot A_{\sigma}^{4} \cdot\left(1-30 \cdot \varphi_{\sigma t}+150 \cdot \varphi_{\sigma t}^{2}-240 \cdot \varphi_{\sigma t}^{3}+120 \cdot \varphi_{\sigma t}^{4}\right)\right)\left.\right|_{t=4 \tilde{\tau}}
\end{gathered}
$$




$$
\begin{aligned}
& \bar{Q}_{6}=\varphi_{\sigma t}^{\prime}-\frac{137}{60} \varphi_{\sigma t}^{\prime \prime}+\frac{15}{8} \varphi_{\sigma t}^{\prime \prime \prime}-\frac{17}{24} \varphi_{\sigma t}^{\iota v}+\frac{1}{8} \varphi_{\sigma t}^{v}-\left.\frac{1}{120} \varphi_{\sigma t}^{v L}\right|_{t=5 \tilde{\tau}}= \\
& \left.\times\left(1-62 \cdot \varphi_{\sigma t}+510 \cdot \varphi_{\sigma t}^{2}-1560 \cdot \varphi_{\sigma t}^{3}+1800 \cdot \varphi_{\sigma t}^{4}-720 \cdot \varphi_{\sigma t}^{5}\right)\right)\left.\right|_{t=5 \tilde{\tau}} \\
& =A_{\sigma} \cdot \varphi_{\sigma t} \cdot\left(1-\varphi_{\sigma t}\right) \cdot\left(1-\frac{137}{60} \cdot A_{\sigma} \cdot\left(1-2 \cdot \varphi_{\sigma t}\right)+\frac{15}{8} \cdot A_{\sigma}^{2} \times\right. \\
& \times\left(1-6 \cdot \varphi_{\sigma t}+6 \cdot \varphi_{\sigma t}^{2}\right)-\frac{17}{24} \cdot A_{\sigma}^{3} \cdot\left(1-14 \cdot \varphi_{\sigma t}+36 \cdot \varphi_{\sigma t}^{2}-24 \cdot \varphi_{\sigma t}^{3}\right)+ \\
& +\frac{1}{8} \cdot A_{\sigma}^{4} \cdot\left(1-30 \cdot \varphi_{\sigma t}+150 \cdot \varphi_{\sigma t}^{2}-240 \cdot \varphi_{\sigma t}^{3}+120 \cdot \varphi_{\sigma t}^{4}\right)-\frac{1}{120} \cdot A_{\sigma}^{5} \times \\
& \bar{Q}_{7}=\varphi_{\sigma t}^{\prime}-\frac{49}{20} \varphi^{\prime \prime}{ }_{\sigma t}+\frac{203}{90} \varphi^{\prime \prime \prime}{ }_{\sigma t}-\frac{49}{48} \varphi_{\sigma t}^{\iota v}+\frac{35}{144} \varphi_{\sigma t}^{v}-\frac{7}{240} \varphi_{\sigma t}^{v l}+ \\
& +\left.\frac{1}{720} \varphi_{\sigma t}^{v u t}\right|_{t=6 \tilde{\tau}}=A_{\sigma} \cdot \varphi_{\sigma t} \cdot\left(1-\varphi_{\sigma t}\right) \cdot\left(1-\frac{49}{20} \cdot A_{\sigma} \cdot\left(1-2 \cdot \varphi_{\sigma t}\right)+\right. \\
& +\frac{203}{90} \cdot A_{\sigma}^{2} \cdot\left(1-6 \cdot \varphi_{\sigma t}+6 \cdot \varphi_{\sigma t}^{2}\right)-\frac{49}{48} \cdot A_{\sigma}^{3} \cdot\left(1-14 \cdot \varphi_{\sigma t}+36 \cdot \varphi_{\sigma t}^{2}-\right. \\
& \left.-24 \cdot \varphi_{\sigma t}^{3}\right)+\frac{35}{144} \cdot A_{\sigma}^{4} \cdot\left(1-30 \cdot \varphi_{\sigma t}+150 \cdot \varphi_{\sigma t}^{2}-240 \cdot \varphi_{\sigma t}^{3}+120 \cdot \varphi_{\sigma t}^{4}\right)- \\
& -\frac{7}{240} \cdot A_{\sigma}^{5} \cdot\left(1-62 \cdot \varphi_{\sigma t}+510 \cdot \varphi_{\sigma t}^{2}-1560 \cdot \varphi_{\sigma t}^{3}+\right. \\
& \left.+1800 \cdot \varphi_{\sigma t}^{4}-720 \cdot \varphi_{\sigma t}^{5}\right)+\frac{1}{720} \cdot A_{\sigma}^{6} \cdot\left(1-126 \cdot \varphi_{\sigma t}+1760 \cdot \varphi_{\sigma t}^{2}-\right. \\
& \left.\left.-8280 \cdot \varphi_{\sigma t}^{3}+16800 \cdot \varphi_{\sigma t}^{4}-15120 \cdot \varphi_{\sigma t}^{5}+5040 \cdot \varphi_{\sigma t}^{6}\right)\right)\left.\right|_{t=6 \tilde{\tau}}
\end{aligned}
$$

The obtained results (59) - (65) can be represented by a single formula (Demidov et al., 2006d).

$$
\bar{Q}_{k}=\left.\bar{r}_{\sigma t} \cdot \sum_{i=1}^{k}\left(A_{\sigma}^{i-1} \cdot P_{k i}\left(\varphi_{\sigma t}\right)\right)\right|_{t=(k-1) \tilde{\tau}}
$$

where $P_{k i}\left(\varphi_{\sigma t}\right) \leftarrow$ polynomials of a degree $i-1$ from $\varphi_{\sigma t}$. The type of the polynomials $P_{k i}\left(\varphi_{\sigma t}\right)$ is shown in Table 2.

Therefore, the delay spectrum obtained on the basis of the mathematical model of the creep process, using the hyperbolic tangent (36) as the normalised delay function is completely determined by the structural coefficient $A_{\sigma}$, depending on a polymer material and the type of the polynomials $P_{k i}\left(\varphi_{\sigma t}\right)$ that do not depend on a polymer material but are the characteristics of the mathematical model itself (Demidov et al., 2007b).

From the analytical form of the delay spectrum approximations $\bar{Q}_{k}$, it is evident that these approximations converge rather quickly to their limiting value $\bar{Q}$ which characterises the stability of the chosen mathematical model of the creep process based on the hyperbolic tangent (36). This means that, with a small permissible error, as the relaxation spectrum its first approximation can be accepted, which is given by (Stalevich et al., 2003)

$$
\bar{Q}_{1}=\left.A_{\sigma} \cdot \varphi_{\sigma t} \cdot\left(1-\varphi_{\sigma t}\right)\right|_{t=\tilde{\tau}}
$$

and depends only on the structural coefficient $A_{\sigma}$ and on the delay function $\varphi_{\sigma t}$ in the form of the hyperbolic tangent (36).

Taking into account that the approximations of the spectrum of delay times $\bar{Q}_{\sigma \tilde{\tau}}$ are obtained by the logarithmic time scale derivatives of the normalised delay function (36), and knowing the type of the delay spectrum the inverse problem can be solved. It is possible to 
Table 2. The type of polynomials $P_{k i}\left(\varphi_{\sigma t}\right)$, included in the formulas for approximations of the relaxation spectra of polymer materials.

\begin{tabular}{llllll}
\hline $\mathrm{ilk}$ & 1 & 2 & 3 & 4 & 5 \\
\hline 1 & 1 & 1 & 1 & 1 & 1 \\
2 & - & $2 \varphi-1$ & $3 \varphi-\frac{3}{2}$ & $\frac{11}{3} \varphi-\frac{11}{6}$ & $\frac{25}{6} \varphi-\frac{25}{12}$ \\
3 & - & - & $3 \varphi^{2}-3 \varphi+\frac{1}{2}$ & $6 \varphi^{2}-6 \varphi+1$ & $\frac{35}{4} \varphi^{2}-\frac{35}{4} \varphi+\frac{35}{24}$ \\
4 & - & - & - & $4 \varphi^{3}-6 \varphi^{2}+\frac{7}{3} \varphi-\frac{1}{6}$ & $10 \varphi^{3}-15 \varphi^{2}+\frac{35}{6} \varphi-\frac{5}{12}$ \\
5 & - & - & - & - & $5 \varphi^{4}-10 \varphi^{3}+\frac{25}{4} \varphi^{2}-\frac{5}{4} \varphi+\frac{1}{24}$ \\
\hline
\end{tabular}

\begin{tabular}{lll}
\hline $\mathrm{i} \mathrm{k}$ & 6 & 7 \\
\hline 1 & 1 & 1 \\
2 & $\frac{137}{30} \varphi-\frac{137}{60}$ & $\frac{49}{10} \varphi-\frac{49}{20}$ \\
3 & $\frac{45}{4} \varphi^{2}-\frac{45}{4} \varphi+\frac{15}{8}$ & $\frac{203}{15} \varphi^{2}-\frac{203}{15} \varphi+\frac{203}{90}$ \\
4 & $17 \varphi^{3}-\frac{51}{2} \varphi^{2}+\frac{119}{12} \varphi-\frac{17}{24}$ & $\frac{49}{2} \varphi^{3}-\frac{147}{4} \varphi^{2}+\frac{343}{24} \varphi-\frac{49}{48}$ \\
5 & $15 \varphi^{4}-30 \varphi^{3}+\frac{75}{4} \varphi^{2}-\frac{15}{4} \varphi+\frac{1}{8}$ & $\frac{175}{6} \varphi^{4}-\frac{175}{3} \varphi^{3}+\frac{875}{24} \varphi^{2}-\frac{175}{24} \varphi+\frac{35}{144}$ \\
6 & $6 \varphi^{5}-15 \varphi^{4}+13 \varphi^{3}-\frac{17}{4} \varphi^{2}+\frac{31}{60} \varphi-\frac{1}{120}$ & $21 \varphi^{5}-\frac{105}{2} \varphi^{4}+\frac{91}{2} \varphi^{3}-\frac{119}{8} \varphi^{2}+\frac{217}{120} \varphi-\frac{7}{240}$ \\
7 & - & $7 \varphi^{6}-21 \varphi^{5}+\frac{70}{3} \varphi^{4}-\frac{23}{2} \varphi^{3}+\frac{110}{45} \varphi^{2}-\frac{31}{180} \varphi+\frac{1}{720}$ \\
\hline
\end{tabular}

obtain the corresponding normalised relaxation function by integrating. That is the basis of the mathematical model of viscoelastic properties (Makarov 2002).

The example of the delay spectrum of a polyester thread 83 tex. is shown in Figure 5. And the corresponding spectrum of the delay times of a polyester fabric made from polyester threads 83 tex. is shown in Figure 6.

Analysing the graphs of the delay times spectra of a polyester thread 83 tex. and polyester fabric made of polyester threads 83 tex., it can be noted that the intensity parameter corresponds to a polyester thread $A_{\sigma}=0,30$, while in a polyester fabric it is much higher $A_{\sigma}=1,45$ which leads to a more pronounced shape of the relaxation spectrum curve (Figure 5., Figure 6.)

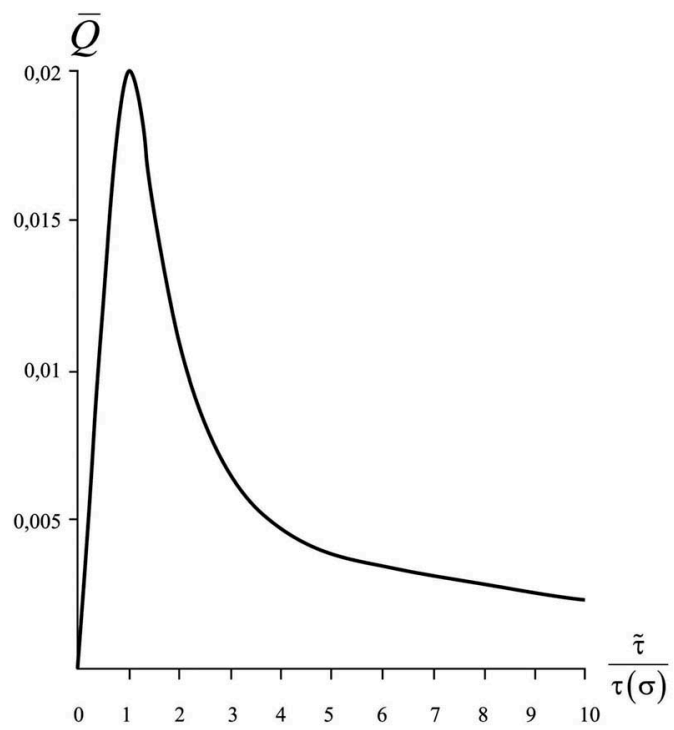

Figure 5. The graph of the spectrum of the delay times of a polyester thread 83 tex., $T=40^{\circ} \mathrm{C}$. 


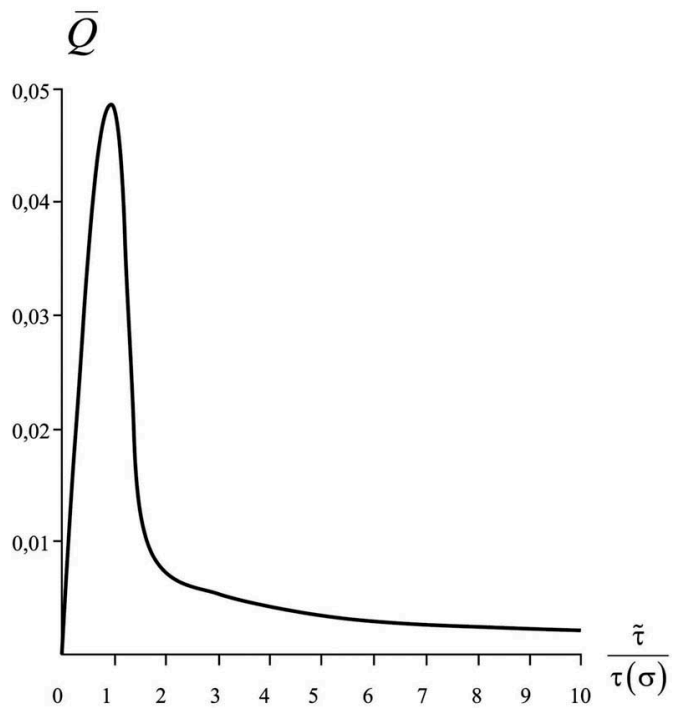

Figure 6. The graph of the spectrum of the delay times of polyester fabric made from polyester threads 83 tex., $T=40^{\circ} \mathrm{C}$.

It should be noted that the modified logarithmic time scale contains the force-time function

$$
\ln \frac{t}{\tau_{\sigma}}=\ln \frac{t}{t_{1}}+\ln \frac{t_{1}}{\tau_{\sigma}}=\ln \frac{t}{t_{1}}+f_{\sigma_{1} \sigma}
$$

reflecting the force-time analogy. Due to this time modification, the obtained delay spectra are generalised, that means, they extend to the entire initial range of non-destructive deformation (Stalevich et al., 2002a).

\section{THE INTERRELATION OF VISCOELASTIC PARAMETERS-CHARACTERISTICS AND THE SPECTRA OF RELAXATION AND DELAY}

As it is obvious from the previous study ( 2 and 3 ), the spectra of relaxation times and delay times are completely determined by the tasks of the mathematical model of relaxation and the mathematical model of creep, respectively, as well as the values of the intensity parameters $A_{\varepsilon}$ and $A_{\sigma}$ of these processes.

The approximations of the spectra of relaxation and delay times converge rather quickly to their limiting values. Therefore, according to its physical meaning, the normalised relaxation function is obtained by integrating the relaxation spectrum which characterises the distribution of particles over relaxation times and is completely determined by the average value of the relaxation times and the value of the structural coefficient $A_{\varepsilon}$. Similar results were achieved by obtaining the delay spectrum from the measured creep processes. If the preference is given to the average relaxation time $\tau_{\varepsilon}$, depending on the deformation $\varepsilon$ and the relaxation spectrum, this physical approach to nonlinear hereditary viscoelasticity can be called geometric (Stalevich et al., 2002b). If the average delay time $\tau_{\sigma}$ and the spectrum of the delay $\overline{\mathrm{Q}}_{\sigma \tilde{\tau}}$ are taken as a basis, i.e. the distribution of the number of particles over the delay time, then this approach can be called the force one (Stalevich et al., 2000).

For complex deformation modes, these approaches correspond to equations of the hereditary type written in a logarithmic time scale as (Makarov et al., 2014c) 


$$
\begin{gathered}
\sigma_{\mathrm{t}}=E_{o} \cdot \varepsilon_{t}+\int_{-\infty}^{\ln t} \varepsilon_{t-s} \cdot \frac{\partial E_{\varepsilon s}}{\partial \ln s} \cdot d \ln s \\
\varepsilon_{\mathrm{t}}=E_{o}^{-1} \cdot \sigma_{t}+\int_{-\infty}^{\ln t} \sigma_{t-s} \cdot \frac{\partial D_{\sigma s}}{\partial \ln s} \cdot d \ln s
\end{gathered}
$$

The equation (69) describes a complex relaxation process when $\varepsilon \neq$ const, and the equation (70) describes a complex creep process when $\sigma \neq$ const.

The first approximations of the relaxation spectrum (Pereborovaet al., 2018e; Makarov et al., 2018b)

$$
\begin{gathered}
\left.\frac{\partial \mathrm{E}_{\varepsilon \mathrm{s}}}{\partial \operatorname{lns}} \cong\left(E_{o}-E_{\infty}\right) \bar{H}_{\varepsilon \tilde{\tau}}\right|_{\tilde{\tau}=s} \\
\left.\frac{\partial \mathrm{D}_{\sigma \mathrm{s}}}{\partial \operatorname{lns}} \cong\left(E_{\infty}^{-1}-E_{o}^{-1}\right) \bar{Q}_{\tilde{\sigma} \tilde{\tau}}\right|_{\tilde{\tau}=s}
\end{gathered}
$$

characterise the spectral meaning of the equations kernels (69) and (70) and, as a result, the preferred type of notation (69) and (70) for practical analysis and calculations. For example, analysis or calculation of the stress-strain diagram by the formula (69) and the recovery deformation process by the formula (70) (Makarov et al., 2015c).

The physical validity of using the equations of state in the form (69) and (70) is also confirmed by the observed proportionality between the logarithm of the statistical relaxation or delay time and the activation energy according to the classical Arrhenius-Boltzmann formula (Demidov et al., 2007c)

$$
\begin{aligned}
& U_{\sigma}=R T \cdot\left(\ln \frac{t_{1}}{\tau_{0}}-\ln \frac{t_{1}}{\tau_{\sigma}}\right) \\
& U_{\varepsilon}=R T \cdot\left(\ln \frac{t_{1}}{\tau_{0}}-\ln \frac{t_{1}}{\tau_{\varepsilon}}\right)
\end{aligned}
$$

where $\tau_{0}$ - the parameter that has the meaning of the duration of the elementary rearrangement

of 'relaxing' particles of the material; $t_{1}=1$ minutes; $\ln \frac{t_{1}}{\tau_{\sigma}}=f_{\sigma_{1} \sigma}$ - force function

where $\tau_{\sigma}$ - delay time; $\ln \frac{t_{1}}{\tau_{\varepsilon}}=f_{\varepsilon_{1} \varepsilon}$ - deformation function where $\tau_{\varepsilon}$ - relaxation time;

$T$ - temperature $T^{\circ} K ; R \stackrel{\tau_{\varepsilon}}{=} k A$ where $k$ is the Boltzmann constant, $A$ - the Avogadro number;

$U_{\sigma}$ - activation energy as a function of stress; $U_{\varepsilon}$ - activation energy as a function of deformation (Romashev et al., 2018).

This proportionality and equality of the initial values of the activation energies correspond to the adequacy of the equations (69) and (70), therefore, to the considered spectra in certain conditions (Blinov et al., 2018). The operability of the equations (69) and (70) means that the considered spectra of the distribution of particles over the relaxation or delay times reflects the nonlinear hereditary viscoelasticity of the considered polymer materials (Krasnyi et al., 2020).

\section{CONCLUSION}

The research showed that the relaxation spectra of polymeric materials are with satisfactory accuracy characterised by their first approximations which are the relaxation kernels, and the subsequent approximations quickly converge to the exact values of the spectra. 
The same can be said about the delay spectra of polymeric materials which are also with satisfactory accuracy characterised by their first approximations, that are the delay kernels, and the subsequent approximations quickly converge to the exact values of the spectra.

The solution to the problem of analytical relationship between the nonlinear hereditary relaxation kernel and the deformation-time relaxation spectrum, as well as between the nonlinear hereditary creep kernel and the force-time creep spectrum, was substantiated by plotting the relaxation function and the delay function in the form of the hyperbolic tangent (2) and (36).

The features of the analysed polymeric materials, from the point of view of the considered mathematical models of viscoelasticity and their properties, include the fact that the relaxation kernel as a function of the logarithm almost coincides with the relaxation spectrum in a similar invariant form with reference to the deformation value, and the delay kernel as a function of time coincides with the delay spectrum in an invariant form with reference to the stress value.

It was found that the relaxation and delay spectra are completely determined by the average value of the relaxation and delay times, and the form of the corresponding curves is determined by the value of the structural coefficients $A_{\varepsilon}$ and $A_{\sigma}$ characterising the intensity of relaxation and creep processes.

According to the physical meaning, the normalised relaxation function and the delay function are obtained by integrating the relaxation spectrum and the delay spectrum, respectively.

The problems of determining the types of the relaxation spectra and delay spectra were reduced to obtaining the coefficients $A_{\varepsilon}$ and $A_{\sigma}$ depending on the structure of polymeric materials, the values of which can be found by express processing of the corresponding experimental sets of relaxation and delay. Therefore, it can be concluded that the relaxation and delay spectra are obtained by experimental data processing.

It should be mentioned that the relaxation and delay spectra are with satisfactory accuracy characterised by their first approximations which are the relaxation kernel and the delay kernel, and the subsequent approximations quickly converge to the limiting values of the spectra.

\section{FUTURE RESEARCH AND ACKNOWLEDGEMENTS}

The study was funded within the framework of the state assignment of the Ministry of Science and Higher Education of the Russian Federation, Project No. FSEZ-2020-0005.

\section{REFERENCES}

Blinov, P., Dvoynikov V., (2018) Rheological and Filtration Parameters of the Polymer Salt Drilling Fluids Based on Xanthan Gum. Journal of Engineering and Applied Sciences, 13: 5661-5664. DOI: $10.36478 / 2018.5661 .5664$

Demidov, A.V., Makarov, A.G., Stalevich, A.M. (2006a) The criteria of optimal selection of mathematical model of textile materials viscoelasticity. Izvestiya Vysshikh Uchebnykh Zavedenii, Seriya Teknologiya Tekstil'noi Promyshlennosti, Vol. 293, No 5, pp. 21-25. eid=2-s2.0-34247548784

Demidov, A.V., Makarov, A.G., Stalevich, A.M., Petrova, L.N., Chelishev, A.M. (2006b) Research of changes of deformation properties of polyester threads depending on twist amount. Izvestiya Vysshikh Uchebnykh Zavedenii, Seriya Teknologiya Tekstil'noi Promyshlennosti, Vol. 292, No 4, pp. 9-13. eid=2-s2.0-33845499474

Demidov, A.V., Makarov, A.G., Stalevich, A.M. (2006c) Methods of computer analysis of viscoelasticity of technical materials. Izvestiya Vysshikh Uchebnykh Zavedenii, Seriya Teknologiya Tekstil'noi Promyshlennosti, Vol. 291, No 3, pp. 13-17. eid=2-s2.0-37849188658

Demidov, A.V., Makarov, A.G., Stalevich, A.M. (2006d) Definition of the mechanical characteristics of textile stuffs at variable temperature. Izvestiya Vysshikh Uchebnykh Zavedenii, Seriya Teknologiya Tekstil'noi Promyshlennosti, Vol. 294, No 6, pp. 15-18. eid=2-s2.0-34250009041

Demidov, A.V., Makarov, A.G., Stalevich, A.M. (2007a) Definition of the computer forecasting trends of deformation properties of textile stuffs. Izvestiya Vysshikh Uchebnykh Zavedenii, Seriya Teknologiya Tekstil'noi Promyshlennosti, Vol. 297, No 2, pp. 14-17. eid=2-s2.0-38849203122 
Demidov, A.V., Makarov, A.G., Stalevich, A.M. (2007b) Predicting the nonlinear hereditary viscoelasticity of polymers. Journal of Applied Mechanics and Technical Physics, Vol. 48, No 6, pp. 897-904. DOI: 10.1007/s10808-007-0114-8

Demidov, A.V., Makarov, A.G., Stalevich, A.M. (2007c) System analysis of viscoelasticity of textile stuffs. Izvestiya Vysshikh Uchebnykh Zavedenii, Seriya Teknologiya Tekstil'noi Promyshlennosti, Vol. 298, No 3, pp. 11-14. eid=2-s2.0-34648822922

Demidov, A.V., Makarov, A.G., Stalevich, A.M. (2009) A version of modeling of nonlinear-hereditary viscoelasticity of polymer materials. Mechanics of Solids, Vol. 44, No 1, pp. 122-130. DOI: 10.3103/ S0025654409010130

Demidov, A.V., Makarov, A.G., Pereborova, N.V., Egorova, M.A. (2017) Forecasting of deformation-relaxation properties of poly amide fabric used to make the canopy. Izvestiya Vysshikh Uchebnykh Zavedenii, Seriya Teknologiya Tekstil'noi Promyshlennosti, Vol. 367, No 1, pp. 250-258. eid=2-s2.0-85033239149

Egorov, I.M., Makarov, A.G., Pereborova,N.V., Kiselev, S.V. (2020) Development of methods of system analysis of viscoelastic-plastic properties of polymeric ropes of marine purpose. Vestnik of Saint Petersburg State University of Technologies and Design. Series 4. Industrial Technologies, No 1, pp. 65-74. DOI 10.46418/2619-0729_2020_1_8

Gorshkov, A.S., Makarov, A.G., Romanova, A.A., Rymkevich, P.P. (2013a) Modelling of directed polymers deformation processes based on the description of the kinetics of supramolecular structures separated by energy barriers. Magazine of Civil Engineering, Vol. 44, No 9, pp. 76-83+103-104. DOI: $10.5862 / \mathrm{MCE} .44 .10$

Krasnyi, V. A., Maksarov, V. V. and Ol't, Y. (2020) "The use of polymer composite materials in the friction nodes downhole oil pumps", Journal of Mining Institute, 211, p. 71. Available at: http://pmi. spmi.ru/index.php/pmi/article/view/5250 (Accessed: 19 August 2020).

Makarov, A.G. (2002) Determining the analytical correlation between the standardized nuclei of relaxation and creep in textile materials. Izvestiya Vysshikh Uchebnykh Zavedenii, Seriya Teknologiya Tekstil'noi Promyshlennosti, Vol. 266, No 2, pp. 13-17. eid=2-s2.0-0036931214

Makarov, A.G., Pereborova, N.V., Egorova, M.A., Wagner, V.I. (2014a) Modeling and forecasting viscoelastic properties of textile materials with a complex structure. Izvestiya Vysshikh Uchebnykh Zavedenii, Seriya Teknologiya Tekstil'noi Promyshlennosti, Vol. 354, No 6, pp. 120-124. eid=2-s2.0 $-84937439497$

Makarov, A.G., Pereborova, N.V., Egorova, M.A., Wagner, M.A. (2014b) Ways of modeling deformation and relaxation properties of textile materials with a complex structure. Izvestiya Vysshikh Uchebnykh Zavedenii, Seriya Teknologiya Tekstil'noi Promyshlennosti, Vol. 351, No 3, pp. 110-115. eid=2-s2 .0-84937410003

Makarov, A.G., Pereborova, N.V., Wagner, V.I., Rymkevich, P.P., Gorshkov, A.S. (2014c) The Basis of Spectral-Temporal Analysis of Relaxation and Deformation Properties of Polymeric Materials in Textile and Ligt Industry. Izvestiya Vysshikh Uchebnykh Zavedenii, Seriya Teknologiya Legkoi Promyshlennosti, Vol. 23, No 1. pp. 24-29.

Makarov, A.G., Slutsker, G.Y., Drobotun, N.V. (2015a) Creep and fracture kinetics of polymers. Technical Physics, Vol. 60, No 2, pp. 240-245. DOI: 10.1134/S1063784215020152

Makarov, A.G., Pereborova, N.V., Wagner, V.I., Vasileva, E.K. (2015b) Development of methodology for the comparative analysis of deformation and relaxation properties of aramid yarns and textile materials based on them. Izvestiya Vysshikh Uchebnykh Zavedenii, Seriya Teknologiya Tekstil'noi Promyshlennosti, Vol. 359, No 5, pp. 48-58. eid=2-s2.0-84971636036

Makarov, A.G., Demidov, A.V., Pereborova, N.V., Egorova, M.A. (2015c) Modeling and prediction of estimated relaxation and deformation properties of the polymer parachute line. Izvestiya Vysshikh Uchebnykh Zavedenii, Seriya Teknologiya Tekstil'noi Promyshlennosti, Vol. 360, No 6, pp. 194-205. eid=2-s2.0-84976560627

Makarov, A.G., Slutsker, G.Y., Gofman, I.V., Vasil'eva, V.V. (2016) Initial stage of stress relaxation in oriented polymers. Physics of the Solid State, Vol. 58, No 4, pp. 840-846. DOI: 10.1134/ S1063783416040132

Makarov, A.G., Pereborova, N.V., Egorova, M.A., Egorov, I.M. (2017a) Quality analysis of deformation-relaxation properties of aramid cords mountain rescue appointments. Izvestiya Vysshikh Uchebnykh Zavedenii, Seriya Teknologiya Tekstil'noi Promyshlennosti, Vol. 368, No 2, pp. 309-313. eid=2-s2.0-85035207042

Makarov, A.G., Pereborova, N.V., Egorova, M.A., Egorov, I.M. (2017b) Mathematical modeling of deformation-relaxation processes polymeric materials in conditions of variable temperatures. Izvestiya Vysshikh Uchebnykh Zavedenii, Seriya Teknologiya Tekstil'noi Promyshlennosti, Vol. 370, No 4, pp. 287-292. eid=2-s2.0-85057142312 
Makarov, A.G., Pereborova N.V., Kozlov A.A., Shvankin A.M. (2018a) Computer-Assisted Prediction and Qualitative Analysis for Polymer Parachute Cords Fibre Chemistry, Vol. 50, No. 3, pp. 239-242. DOI 10.1007/s10692-018-9968-1

Makarov, A.G., Pereborova, N.V., Vagner, V.I., Egorova, M.A., Klimova, N.S. (2018b) Spectral Analysis of Viscoelastic Creep of Geotextiles. Fibrie Chemistry, Vol. 50, No 4, pp. 378-382. DOI 10.1007/ s10692-019-09993-4

Makarov, A.G., Busygin, K.N. (2020) System analysis of functional properties of special application aramid materials. Vestnik of Saint Petersburg State University of Technologies and Design. Series 4. Industrial Technologies, No 2, pp. 84-94. DOI: 10.46418/2619-0729_2020_2_10

Pereborova, N.V., Makarov, A.G., Egorova, M.A., Kozlov, A.A., Konovalov, A.S. (2018a) Methods of simulation and comparative analysis of shadow and deformation-reducing properties of aramide textile materials. Izvestiya Vysshikh Uchebnykh Zavedenii, Seriya Teknologiya Tekstil'noi Promyshlennosti, Vol. 375, No 3, pp. 253-257. eid=2-s2.0-85059766891

Pereborova, N.V., Makarov, A.G., Kozlov, A.A., Vasil'eva, E.K. (2018b) Development of Integral Optimality Criteria for Mathematical Modeling of Relaxation/Recovery Processes in Polymer Textile Materials. Fibrie Chemistry, Vol. 50, No 4, pp. 306-309. DOI 10.1007/s10692-019-09981-8

Pereborova, N.V., Makarov, A.G., Egorova, M.A., Klimova, N.S. (2018c) Methods of increasing the competitiveness of domestic aramid textile materials based on complex analysis of their functional properties. Izvestiya Vysshikh Uchebnykh Zavedenii, Seriya Teknologiya Tekstil'noi Promyshlennosti, Vol. 378, No 6, pp. 267-272. eid=2-s2.0-85072335464

Pereborova, N.V., Demidov, A.V., Makarov, A.G., Klimova, N.S., Vasileva, E.K. (2018d) Methods of mathematical modeling and qualitative analysis of relaxation-deformation processes of aramide textile materials. Izvestiya Vysshikh Uchebnykh Zavedenii, Seriya Teknologiya Tekstil'noi Promyshlennosti, Vol. 374, No 2, pp. 251-255. eid=2-s2.0-85056451197

Pereborova, N.V., Demidov, A.V., Makarov, A.G., Klimova, N.S. (2018e) Modeling of DeformationRelaxation Processes of Aramid Textile Materials - the Foundation for Analyzing Their Operational Properties. Fibre Chemistry, Vol. 50, No. 2, pp. 104-107. DOI 10.1007/s10692-018-9941-z

Pereborova, N.V., Makarov, A.G., Vasil'eva, E.K., Shvankin, A.M., Egorov, I.M. (2019a) Mathematical Modeling and Computed Prediction of Viscoelastic Creep in Geotextile Nonwoven Fabrics. Fibre Chemistry, Vol. 50, No. 6, pp. 487-490. DOI 10.1007/s10692-019-10015-6

Pereborova, N.V., Makarov, A.G., Egorova, M.A., Kozlov, A.A. (2019b) Mathematical Modeling and Comparative Analysis of Deformation/Recovery Properties and Shrinkage of Aramid Textile Materials. Fibre Chemistry, Vol. 50, No 5, pp. 468-472. DOI 10.1007/s10692-019-10010-x

Pereborova, N.V., Makarov, A.G., Egorova, M.A., Klimova, N.S. (2019c) Improving the Competitiveness of Aramid Textile Materials Based on Mathematical Modeling and Analysis of Their Performance Properties. Fibre Chemistry, Vol. 50, No 6, pp. 569-572. DOI 10.1007/s10692-019-10030-7

Pereborova, N.V., Makarov, A.G., Shvankin, A.M., Egorova, M.A., Abramova, I.V. (2020a) Modeling and Qualitative Analysis of Creep Processes of Geotextile Nonwovens -A Foundation for Enhancing their Competitiveness. Fibre Chemistry, Vol. 51, No. 5, pp. 397-400. DOI 10.1007/s10692-020-10119-4

Pereborova, N.V., Makarov, A.G., Shvankin, A. M., Egorova, M. A., Korobovtseva, A.A. (2020b) Predicting Creep and Deformation and Recovery Processes of Geotextile Nonwovens. Fibre Chemistry, Vol. 51, No. 5, pp. 401-403. DOI 10.1007/s10692-020-10120-x

Pereborova, N.V., Makarov, A.G., Egorova, M.A., Egorov, I.M. (2020c) Methods of Modeling and Computer-Aided Prediction of Relaxation of Medical-Purpose Textile Elastomers. Fibre Chemistry, Vol. 51, No. 6, pp. 467-470. DOI 10.1007/s10692-020-10136-3

Pereborova, N.V., Makarov, A.G., Egorova, M.A., Egorov, I.M. (2020d) Methods modeling and Computer-Aided Prediction of Strain and Relaxation Processes of Medical-Purpose Textile Elastomers. Fibre Chemistry, Vol. 51, No. 6, pp. 471-474. DOI 10.1007/s10692-020-10137-2

Pereborova, N.V., Makarov, A.G., Kiselev, S.V., Egorov, I.M. (2020e) System analysis of relaxation processes of polymeric textile materials of special purpose. Vestnik of Saint Petersburg State University of Technologies and Design. Series 4. Industrial Technologies, Vol. 47, No 1, pp. 53-64. DOI 10.46418/ 2619-0729_2020_1_7

Pereborova, N.V., Wagner, V.I., Kiselev, S.V., Kozlov, A.A. (2020f) Computer forecasting of relaxation processes of polymeric textile materials. Vestnik of Saint Petersburg State University of Technologies and Design. Series 4. Industrial Technologies, No 1, pp. 89-100. DOI 10.46418/2619-0729_2020_1_12

Pereborova, N.V. (2020g) Application of criteria for qualitative evaluation of relaxation properties of polymeric textile materials for evaluation of their functionality. Vestnik of Saint Petersburg State University of Technologies and Design. Series 4. Industrial Technologies, No 1, pp. 101-110. DOI 10.46418/ 2619-0729_2020_1_13 
Romashev, A.O., Aleksandrova, T.N., Aleksandrov, A.V. (2018) Modelling of the relaxation properties of petroleum-bitumen suspension. Information, No 20, Vol. 9, pp. 6595-6603

Rymkevich, P.P., Romanova, A.A., Golovina, V.V., Makarov, A.G. (2013) The energy barriers model for the physical description of the viscoelasticity of synthetic polymers: Application to the uniaxial orientational drawing of polyamide films. Journal of Macromolecular Science, Part B: Physics, Vol. 52, No 12, pp. 1829-1847. DOI: 10.1080/00222348.2013.808906

Stalevich, A.M., Makarov, A.G. (2000) Determining the inherent viscoelastic relaxation spectrum for synthetic filaments. Izvestiya Vysshikh Uchebnykh Zavedenii, Seriya Teknologiya Tekstil'noi Promyshlennosti, Vol. 255, No 3, pp. 8-12. eid=2-s2.0-0034436083

Stalevich, A.M., Makarov, A.G. (2002a) Forecasting the deformation recovery process and the reverse relaxation in polymer materials. Izvestiya Vysshikh Uchebnykh Zavedenii, Seriya Teknologiya Tekstil'noi Promyshlennosti, Vol. 267, No 3, pp. 10-13. eid=2-s2.0-0038128574

Stalevich, A.M., Makarov, A.G., Saidov, E.D. (2002b) Elastic components in the stress/strain curve for a synthetic fibre yarn. Izvestiya Vysshikh Uchebnykh Zavedenii, Seriya Teknologiya Tekstil'noi Promyshlennosti, Vol. 268, No 4-5, pp. 15-18. eid=2-s2.0-0037742684

Stalevich, A.M., Makarov, A.G., Saidov, E.D. (2003) Relaxation spectrometry of synthetic yarns. Izvestiya Vysshikh Uchebnykh Zavedenii, Seriya Teknologiya Tekstil'noi Promyshlennosti, Vol. 270, No 1, pp. 16-22. eid=2-s2.0-2642532049 